\title{
LUPUS ERITEMATOSO NEONATAL: REPORTE DE CASO DE LACTANTE CONCEBIDA POR INSEMINACIÓN ARTIFICIAL Y MADRE CON ARTRITIS REUMATOIDEA
}

\section{Lupus eritematoso neonatal: Report of the infant case conceived by artificial insemination and mother with rheumatoid arthritis}

\author{
Daisy M. Blanco M.D. ${ }^{1 *}$, Marta Miniño M.D. ${ }^{1 * *}$, Chrismel C. Leguisamón M.D. ${ }^{1 * * *}$, \\ Nicole A. Melo M.D. ${ }^{* * * *}$
}

Recibido: febrero 5, 2019 • Aprobado: abril 25, 2019

Cómo citar: M. Blanco D, Miniño M, C. Leguisamón C, A. Melo N. Lupus eritematoso neonatal: reporte de caso de lactante concebida por inseminación artificial y madre con artritis reumatoidea. cysa [Internet]. 26 de julio de 2019 [citado 26 de julio de 2019];3(2): 71-76. Disponible en: https://revistas.intec.edu.do/index.php/cisa/article/view/1481

\section{Resumen}

El Lupus Eritematoso Neonatal es una enfermedad de origen autoinmune caracterizada por rash cutáneo transitorio, bloqueo cardíaco congénito permanente, función hepática anormal con o sin enfermedad biliar y compromiso hematológico asociado a la presencia de autoanticuerpos maternos contra la ribonucleoproteinas solubles $(\mathrm{SSB} / \mathrm{La}$, SSA/Ro y Anti-RNP). Se presenta el caso de una niña de cinco meses de edad con hallazgos clínicos e histopatológicos de Lupus eritematoso neonatal. Es una condición que no suele dejar secuelas, aunque se han reportado casos de atrofia cutánea e hiperpigmentación.

Palabras clave: Lupus eritematoso neonatal; ojos de mapache; anti-Ro; anti-La; autoinmune.

\begin{abstract}
Neonatal Lupus Erythematosus is an autoimmune disease characterized by transitory cutaneous rash, congenital heart block, abnormal liver function test with or without cholestasis and hematologic features associated to anti-Ro and anti-La autoantibodies. A 5-month-old female is brought to the hospital with clinical and histopathology findings of Neonatal lupus. This medical condition does not leave long term physical damage, however there have been cases reported with cutaneous atrophy and hyperpigmentation.
\end{abstract}

Keywords: Neonatal Lupus Erythematosus; Raccoon Eyes; anti-Ro; anti-La; autoimmune.

Correo-e de los autores:

*dmbf1971@hotmail.com

**dermatologyca@gmail.com

*** chrismel.leguisamon@gmail.com

**** nicolemelomendieta@gmail.com 


\section{Introducción}

El Lupus Eritematoso Neonatal es una enfermedad autoinmune infrecuente, con una incidencia estimada de 1/10,000 - 20,000 nacidos vivos y un predominio en el sexo femenino. Causado por la transferencia pasiva a través de la placenta de anticuerpos maternos SSB/La, SSA/Ro y menos frecuente Anti-RNP.

El pasaje transplacentario de los anticuerpos maternos es clave para el desarrollo de este síndrome; aproximadamente el $50 \%$ de los casos consiste en lesiones de la piel, que desaparecen sin dejar cicatriz, coincidiendo con la depuración de esos anticuerpos en los primeros meses. En efecto, los pacientes son seronegativos cuando son investigados después de los 6 meses de vida, mientras que su perfil de autoanticuerpos es muy similar o igual al de la madre inmediatamente después del parto. El tratamiento del Lupus Neonatal es sintomático y específico de cada órgano afectado.

\section{Caso clínico}

Paciente femenina de 5 meses de edad, fototipo III de Fitzpatrick, producto de madre GeSTA 2, PARA 0, ABORTO 1 concebida vía Fecundación In Vitro, procedente y residente en Santo Domingo, quien es traída a consulta por su madre por dermatosis que afecta cara desde el nacimiento, y dos meses después se extiende a tronco, extremidades superiores e inferiores, asintomática. Dermatosis constituidas en cara por múltiples máculas eritematosas, algunas violáceas, de límites mal definidos e irregulares, de predominio periocular con apariencia de "ojos de mapache" (Figura 1). En el tronco y las extremidades se observan placas eritematosas, ligeramente infiltradas, de bordes irregulares, algunas policíclicas, otras anulares, que confluyen formando placas de tamaño variable (Figura 2).

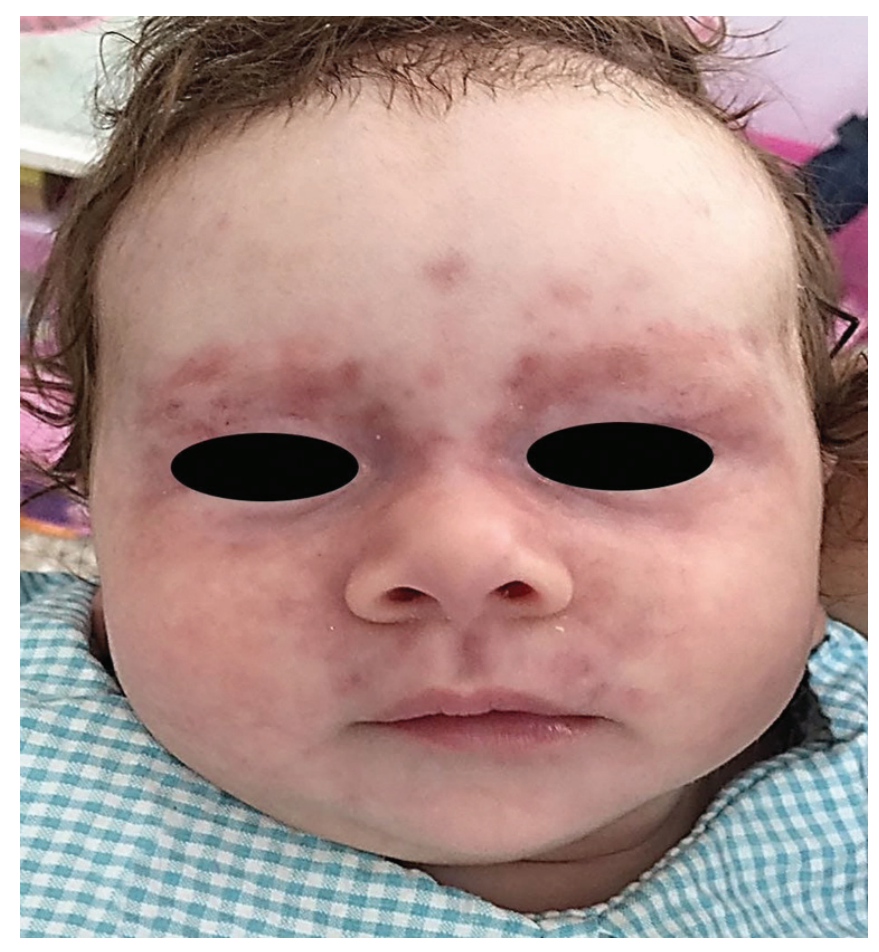

Figura 1. Lupus neonatal. Ojos de mapache

Fuente: archivo personal de los autores. Fotos de la paciente.

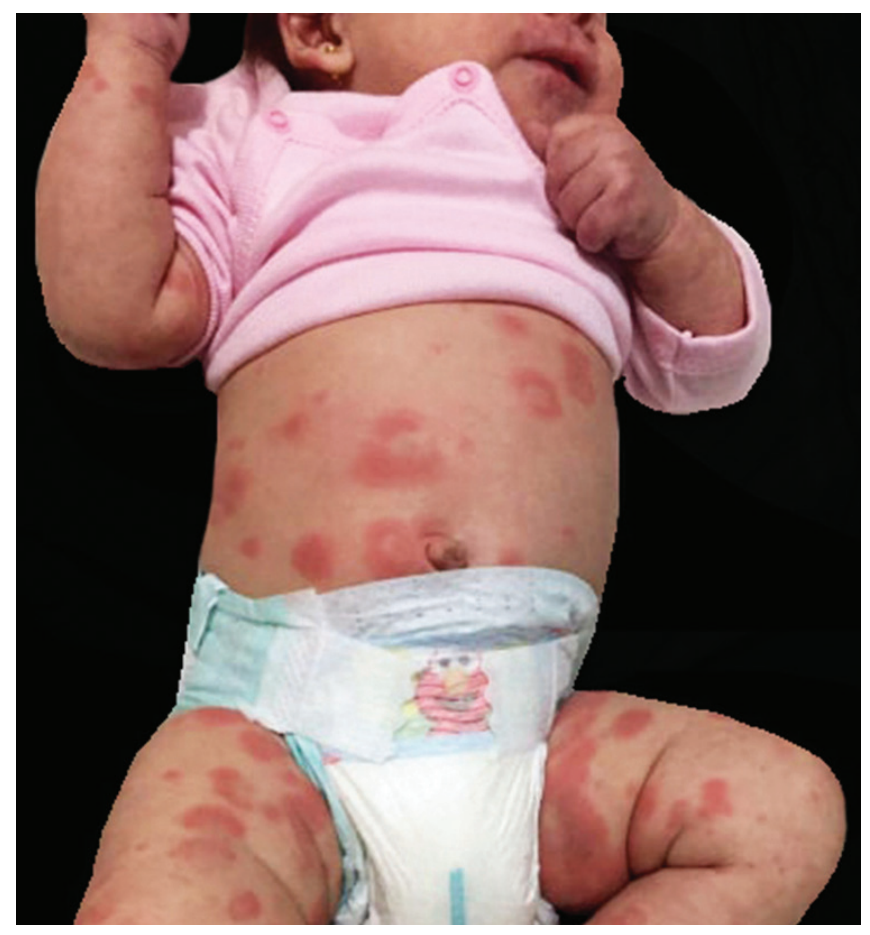




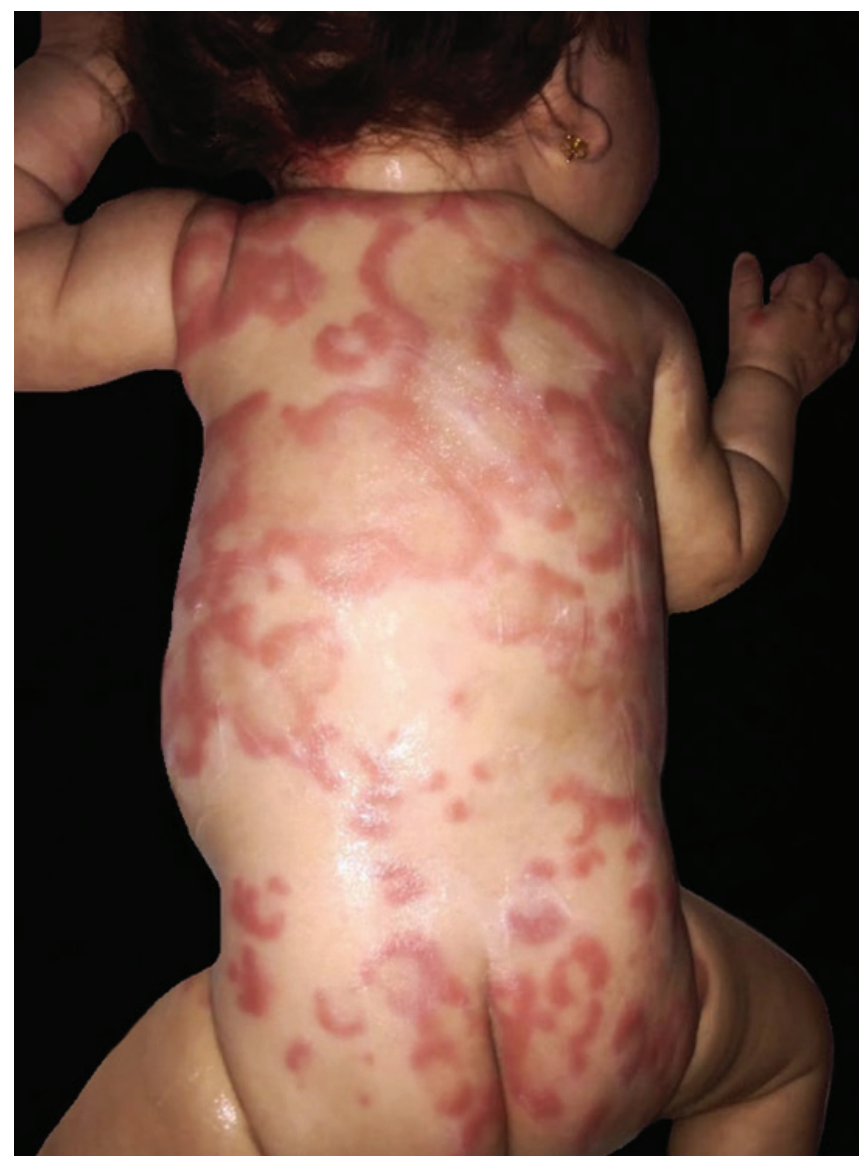

Figura 2. Placas eritematosas difusas anulares y policíclicas en tronco, espalda y extremidades superiores e inferiores

Fuente: archivo personal de los autores. Fotos de la paciente.

Semanas previas a su evaluación por el Departamento de Dematología-pediátrica la paciente estuvo ingresada por dermatosis que afecta tronco, extremidades superiores e inferiores, con el diagnóstico de urticaria, la cuales se solucionaron con el uso de corticoesteroides.

Antecedentes heredo-familiares: madre con artritis reumatoide de 10 años de evolución. Padre con hipertensión arterial, varicocele e infertilidad más azoospermia.

El mismo día de su valoración dermatológica se le realiza biopsia por punch, la cual histopatológicamente reporta dermatitis perivascular y perianexial más vasodilatación (Figura 3), descartar Lupus Neonatal.

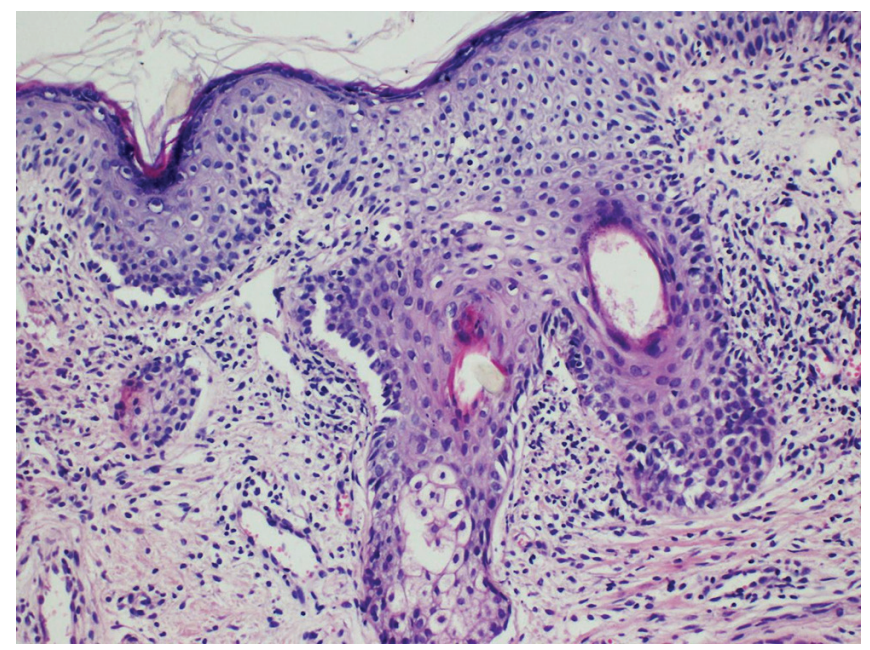

Figura 3. Se observa infiltrado inflamatorio difuso perivascular y perianexial

Fuente: archivo personal de los autores.

Se le realizan a la paciente estudios analíticos que reportan: Hb 10.20g/dl, HTC 29.40 \%, VCM $80.11 \mathrm{fL}$, HCM 27.79 pg. Glicemia $78 \mathrm{mg} / \mathrm{dl}$, VSG $18 \mathrm{~mm} / 1 \mathrm{~h}$, AST $156.50 \mathrm{u} / \mathrm{l}$, ALT 121.20 u/l. ANA positivo 1:160, Anti-Ro positivo, Factor Reumatoide 18.40, Complementos C3 disminuido (60mg/dl) C4: -3mg/dl, TPO: 6.80mg/dl, AntiDNA: negativo, Anti-SM: negativo, Anti-RNP: negativo, Anti-JO-1: negativo, SCL-70: negativo. Examen de orina dentro de límites normales. Sonografía abdominal, evaluación cardiovascular y oftalmológica sin evidencias patológicas.

Se le realizaron analíticas a la madre que reportan: Hb $11.10 \mathrm{~g} / \mathrm{dl}$, HTC 32.0 \%, VCM 86.49fL, HCM 27.27pg, PQT 670k/ul, ANA positivo, Anti-Ro positivo, Factor Reumatoide: 401, AntiCCP:417.90, VSG: 48, PCR: 2.55, TPO: 214.35, Anti DNA: negativo, Anti-SM: negativo, AntiRNP: negativo, Anti-JO-1: negativo, SCL-70: negativo, C3: 157, C4: 32.60, ALT: 10, AST: 13.8, creatinina: 0.63 , microalbumina 24 horas: 8.03 $\mathrm{mg} / 24 \mathrm{~h}$, proteínas en orina $24 \mathrm{hrs} 98.8 \mathrm{mg} / 24 \mathrm{~h}$.

Se decide iniciar tratamiento por el Departamento de Dermatología-Pediátrica con protector solar de amplio espectro diario cada dos horas y chequeos 
rutinarios multidisciplinarios. Seis meses después, paciente acude a consulta nuevamente con notable mejoría de su cuadro clínico (Figura 4). A la exploración física, se observa atelectasias en la parte externa de ambos párpados superiores. Se decide continuar con igual medida terapéutica.
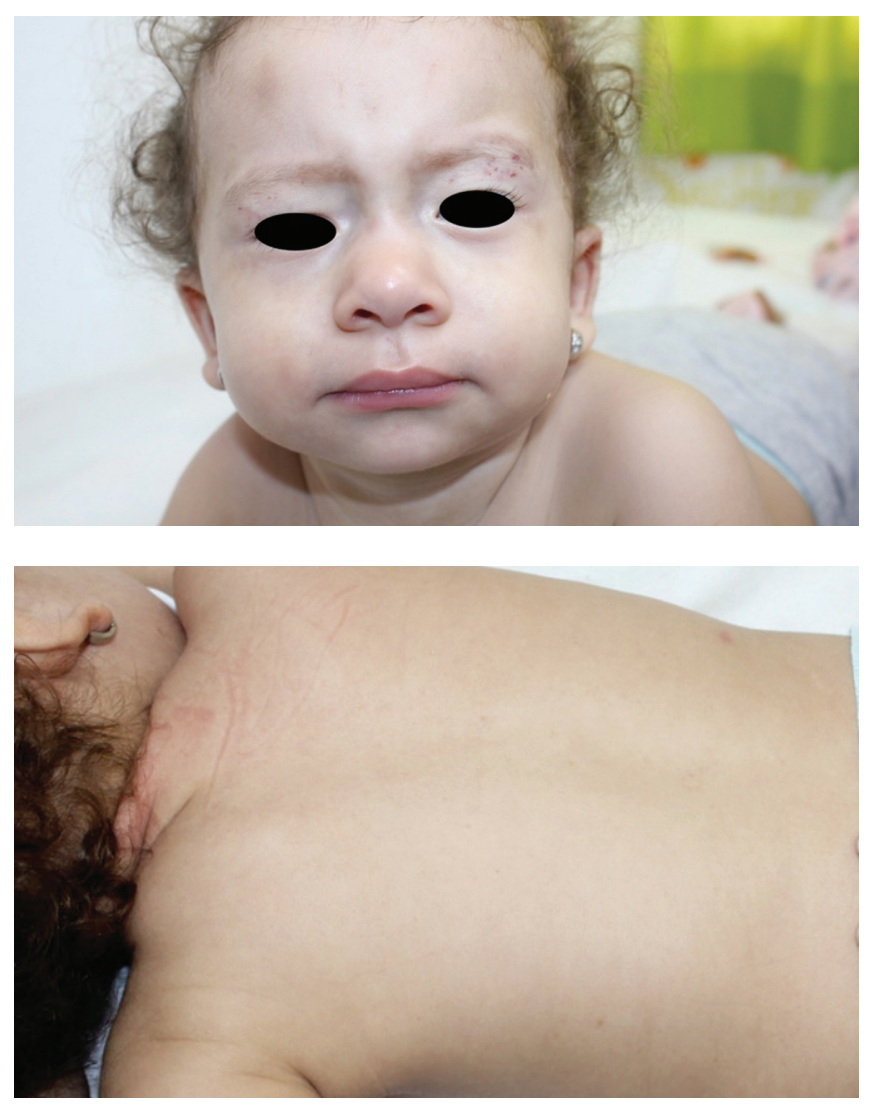

Figura 4. Resolución de lesiones en tronco y leve telangiectasia en área periocular

Fuente: archivo personal de los autores. Fotos de la paciente.

\section{Discusión}

El Lupus Eritematoso Neonatal afecta aproximadamente del $10 \%$ al $20 \%$ de los nacidos de madre con anticuerpos anti-Ro y anti-La que no han sido diagnosticadas de Lupus, síndrome de Sjögren o síndrome autoinmune indiferenciados con anticuerpos circulantes ${ }^{1,12}$. Los anticuerpos maternos anti-Ro y anti-La son transferidos a través de la placenta ${ }^{2,7,11}$. Los anticuerpos anti-Ro se encuentran usualmente en el síndrome de Sjögren, pero se pueden encontrar en un $30 \%$ en pacientes con lupus eritematoso sistémico con características cutáneas ${ }^{3,6}$. Aproximadamente el $50 \%$ de los bebés presentan manifestaciones cutáneas a las 6 semanas de edad. ${ }^{13}$ Sin embargo, el $23 \%$ de los bebés afectados presenta manifestaciones al nacimiento, especialmente las manifestaciones cardíacas y ocasionalmente las cutáneas. Las lesiones son comúnmente localizadas en áreas foto-expuestas, particularmente en la cabeza, cuello y extensores de extremidades superiores. Se ha descrito en un $5 \%$ de los pacientes lesiones en palmas de la mano y plantas de los pies, así como en la vulva. El eritema en la cara, especialmente en región periorbitaria (ojos de mapache), y las lesiones anulares casi siempre se consideran diagnósticas de lupus. Las lesiones discoides, parches atróficos descamativos y telangiectasias también se pueden presentar. ${ }^{1,9}$

En el caso descrito resaltan manifestaciones cutáneas en la cara en forma de ojos de mapache desde el nacimiento ${ }^{1}$. Por otra parte, nos llama la atención manifestaciones correspondientes a artritis reumatoides que presentaba la madre al momento de la evaluación, lo cual nos lleva a sospechar e indagar sobre una posible patología autoinmune. Es por esto que se procede a realizar un perfil inmunológico tanto en la paciente como en la madre, confirmando el diagnóstico de Lupus Eritematoso Neonatal asociado a anticuerpos maternos.

A parte de las manifestaciones cutáneas se describen manifestaciones extra-cutáneas como el bloqueo cardíaco congénito que ocurre en un $15 \%$ a un $30 \%$ de los pacientes afectados. Otra complicación sistémica envuelve el hígado en un $10 \%$ a un $26 \%$ (hepatomegalia, anormalidades de la función hepática, enfermedad colestásica y fallo hepático), esplenomegalia y linfadenopatías 5 . También enfermedades hematológicas en un $27 \%$ de los infantes afectados incluyen, leucopenia, trombocitopenia y anemia. ${ }^{1}$

Hallazgos histopatológicos en el lupus neonatal se parece al del lupus cutáneo subagudo, con un infiltrado linfocitico rodeando el plexus vascular 
superficial y las estructuras anexas que puede extenderse hacia la dermis y el tejido subcutáneo., ${ }^{5,8,15} \mathrm{La}$ biopsia tomada de nuestro paciente presenta signos de inflamación, como disperso infiltrado neutrofílico tanto perivascular y perianexial. ${ }^{8}$

El tratamiento del lupus neonatal es específico de cada órgano y depende de su sintomatología. Infantes con lupus neonatal quienes se presentan con anormalidades cutáneas, hematológicas y función hepática anormal usualmente tienen una resolución de los signos y síntomas en 4 a 6 meses, con la desaparición de los anticuerpos maternos. ${ }^{1,2,4}$ Esto ocurrió con nuestra paciente. Se utilizaron medidas de soporte como evitar la exposición solar, así como la aplicación de protector solar de amplio espectro. Corticoesteroides tópicos de baja a mediana potencia se pueden utilizar en algunos pacientes con lesiones cutáneas, pero usualmente las lesiones desaparecen espontáneamente ${ }^{1,10}$. Sin embargo, se han reportado casos de atrofia e hiperpigmentación con el uso de los mismos. ${ }^{4,14}$

\section{Conclusión}

Luego de estudiar el caso presentado llegamos a la conclusión de que la asociación principal para el desarrollo de esta patología, Lupus Neonatal, es la transferencia de anticuerpos transplacentarios de la madre al hijo. En este caso, principalmente nos llama la atención la historia tanto de la paciente como de la madre, pues fue concebida vía fecundación in vitro y la madre desconocía su estado inmunológico hasta el cuadro clínico presentado por su hija. El tratamiento en este caso se basa en estricta foto protección y evaluaciones rutinarias de acuerdo a la sintomatología de la paciente.

\section{Bibliografía}

1. Amy S, Paller AJM, Hurwitz, S. Clinical Pediatric Dermatology: a textbook of skin disorders of childhood and adolescence. Philadelphia: Elsevier Saunders; 2011.
2. Chang, C. Neonatal autoimmune diseases: a critical review. J autoimmune. 2012;38: J223-J238.

3. Kumar Y, Bhatia A. Detection of antinuclear antibodies in SLE. Methods Mol Biol. 2014; 1134: 37-45.

4. Savino F, Viola S, Tarasco V, Locatelli E, Ricagni A, Coppo P. Neonatal lupus erythematosus: a cutaneous cases based update. Ital J Pediatrics. 2016;42: 1 .

5. Zandile S, Carol H, Komala P. Neonatal lupus erythematosus or Sweet syndrome? JAAD. 2018; 4(8): 780-3.

6. Kristiana G,Sandeep C, Amolak B.. Neonatal Lupus Erythematous associated with breast milk autoantibodies. JAAD. 2007;56(2): AB155.

7. Neidenbach P J, Sahn. E E. La (SS-B)-positive neonatal lupus erythematosus: Report of a case with unusual features. JAAD. 1993;29(5), Part 2: 848-52.

8. Raymond B, Crowson A, Magro C, Piepkorn M. Dermatopathology. (3rd ed.). Unites States of America: McGraw-Hill; 2010.

9. Thornton C, Eichenfield L, Shinall E, Siegfried E, Rabinowitz L, Esterly N, Luchy A, Friedlander S. Cutaneous telangiectases in neonatal lupus erythematosus. JAAD. 1995;33(1):19-25.

10. Rattanavalai Chantor, MD. Henry W Lim, MD. Tor A. Shwayder, MD. Photosensitivity disorders in children. JAAD. 2012;67(6): 1113. e1-1113.e15.

11. Heelan K, Watson R, Collins SM . Neonatal lupus syndrome associated with ribonucleoprotein antibodies: NCBI. 2013 julio-agosto; 30(4): 41623. doi: 10.1111 / pde.12088. Epub 2013 Feb 22.

12. Lee LA. Transient autoimmunity related to maternal autoantibodies: neonatal lupus: NCBI. 2005 Apr;4(4):207-13. Epub 2004 Dec 8. 
13. Aranegui B, Batalla A, Flórez A, Torre de la C, Dávila P. A case of neonatal lupus erythematosus with cutaneous and cardiac involvement: JAAD. 2011; 64(2), Supplement 1: AB137.

14. Briggs L, Levy R, Lara-Corrales I, Silverman E, Pope E. Cutaneous scarring in neonatal lupus: A retrospective cohort study. JAAD. 2018; 79(3), Supplement 1: AB96.
15. Kaneko F, Tanji O, Hasegawa T, Ohto H, Yamazaki K. Neonatal lupus erythematosus in Japan. JAAD. 1992;26(3): 397-403. 